\title{
Organic Solar Cells-A Review on Revolution in the Photovoltaic Research
}

\author{
Rupanjali Meena, Praveen Kumar Jain \\ Department of Electronics and Communication Engineering, Swami Keshvanand Institute of Technology, \\ Management \& Gramothan, Jaipur, Rajasthan (INDIA) \\ Email: meenarupanjali03@gmail.com \\ Received 06.02.2021, Received in revised form 18.03.2021, Accepted 26.03.2021
}

\begin{abstract}
Organic Solar Cell is the third-generation technology in the field of photovoltaic and a great achievement in usage of renewable energy in such an efficient and reliable manner. This technology aims at providing electricity at lower cost than first- and second-generation solar technologies. This article summarizes the efficiency limitations as well as long term reliability rate. Organic photovoltaic have achieved efficiencies near $11 \%$, but efficiency limitations as well as long term reliability remain significant barriers. It will also highlight the future research challenges. In organic solar cell there is low consumption of material per area which results in easy processing of organic semiconductor offering a huge potential for low cost large area solar cells. From a recent search by the researchers it is being observed that increases in efficiency can be achieved by using luminescent acceptor molecules. According to them, the wavelength at which the sunlight is absorbed can be 'adjusted' via the macro-modules used. For example, an office window coated with organic solar cells that absorb the red and infrared spectrum will not only screen out thermal radiation but at the same time will generate electricity. Hence the future research should concentrate on preventing the exciton from decaying which means increase its excitation lifetime. Excitons can decay by emitting light (luminescence) or heat. Multijunction cells are a way to achieve the efficiencies needed. Thin film PV technologies provide numerous ways to reduce manufacturing cost by using high-throughput manufacturing paradigms that don't require handling of individual silicon wafers. Despite the success of entrenched thin-film PV technologies, organic PV cells attract attention because the electronic and optical properties of organic materials can be changed by altering the molecular structure of the materials.

Therefore here in this article we will emphasise on the efficiency and stability rate as well as how can we get high-throughput by using various organic materials together and what are its atmospheric processing.
\end{abstract}

Keywords: Third Generation Technology, Photovoltaics, Efficiency, Stability, Solar Cell Technologies.

\section{INTRODUCTION}

Organic solar cell is the fastest growing technology in this era because of having few withstanding qualities such as usage of organic materials to produce energy from light, flexible, lightweight, highly tuneable \& can be used for both commercial and residential purpose, have less toxicity, safe to install resulting in less damage on environment. They are also unparalleled in the number of times that they can pay back the energy used in their manufacture.

It is still a big task to overcome all barriers to develop a organic solar cells with low cost but at the same time providing high efficiency and stability rate resulting less chances to harm the environment. All existing technology were discarded because increase in the rate of global warming. Extra attention has been given to photo generation, doping effects and improvised learning of photovoltaics mechanism.

Solar Radiation is a general term for the electromagnetic radiation emitted by the sun. This solar radiation can be seized and changed to useful form of energy using variety of technologies. The Earth revolves around the sun in an elliptical orbit [1][2]. When the Sun is nearer to the Earth; the earth's surface receives a little more solar energy. The $23.5^{\circ}$ tilt in the Earth's axis of rotation is a more of a significant factor in determining the amount of sunlight striking the Earth at a particular location.

Radiation data for solar electric systems can be represented by kilowatt-hours per square meter $\left(\mathrm{kWh} / \mathrm{m}^{2}\right)$. Whereas direct estimate can also be expressed as watts per square meter $\left(\mathrm{W} / \mathrm{m}^{2}\right)$.

\section{ORGANIC PV SOLAR CELL SYSTEM}

Earlier solar cell technology comprises of thin silicon wafers which used to transfer sun energy to electric energy, whereas the current photovoltaic's technology is based on the principle of electron hole creation in each cell composed of two different layers (p-type \& n-type materials) of a semiconductor material. The different types of materials applied for photovoltaics solar cells include in the form of silicon, cadmium-telluride, and copper-indium-gallium. Such polymeric OPV material the energy levels of highest occupied molecular orbits (HOMOs) \&lowest unoccupied molecular orbits (LOMOs) are analogous to the 
respective valence \&conduction band of conventional inorganic semiconductor [3][4].

\section{ADVANCES IN SOLAR CELL TECHNOLOGY}

From a very long period of time researchers have been finding ways to enhance the stability, efficiency \& most importantly the life-line of solar cell technology i.e. cost effectiveness. The average solar cell is $15 \%$ efficient which concludes that $85 \%$ of the sunlight which hits solar cell does not make electricity. As a result scientists are continuously putting efforts to test new technology to store light and process its conversion.

\section{ADVANCES IN SOLAR CELL MANUFACTURING}

The only way to make solar panels more powerful is to go bigger. But then having a giant solar panel, one way to create a more powerful module in the same sized footprint is to switch to bigger silicon wafers. These silicon wafers are the building blocks of crystalline silicon solar cells which string together to become complete solar panels. The bigger the wafer, the more power it can generate because of its larger surface area.

Till date, most of the OSCs device specifications are carried under common laboratory conditions with use of solar simulators \& under nitrogen atmosphere. Anyways, these devices will be utilized under different conditions fitting to applications, such as under atmosphere, greater than one-sun illumination, indoor low-light illumination $\&$ under water. Moving over, apart from all existing techniques in optimizing molecular packing in active-layer films, the selective removal of electron acceptors near the top electrode facilitates the realization of highly durable OSCs that can even function under water without encapsulation.

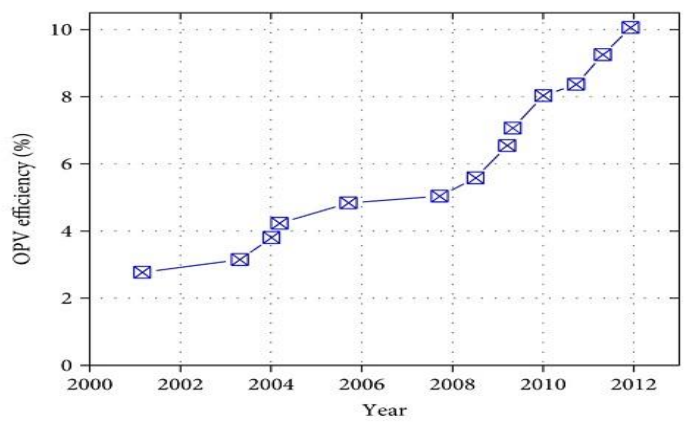

Fig.1 Yearly development of laboratory organic solar cell PCEs.

\section{APPLICATION}

There are numerous applications of OSCs, but one of the most practical \& important use of solar energy is to use the combination of solar collector which produces electricity and hot water. There is this Swedish company SOLARUS. The measured output efficiency is $56 \%$, plus the hot water, which is ideal for businesses that use a lot of hot water or families with many members or restaurants etc. Few applications listed below:

Solar Skin Design: The MIT start-up has created a "solar skin" product that makes it possible for solar panels to match the appearance of a roof without interfering with panel efficiency or production.

Solar Powered Roads: Last Summer in America a particular test was carried out for solar powered pavement tech. These roadways are indicated to generate clean energy, but they were equipped with LED bulbs that can light roads at night \&have the thermal heating capacity to melt snow during winter weather [5][6].

- Wearable Solar: This term isn't any new to us as solar-powered watches and other gadgets are out in the market for several years. Here the only changes are tiny solar panels can now be switched into the fabric of clothing. It can also be used for various home products such as window curtains and heated car seats.

Solar Batteries ;(an innovation in solar storage): The concepts of off-grid solar\& solar plus storage have gained popularity in U.S. markets. Solar storage is still a fairly expensive product in 2019, but a surge in demand from shoppers is expected to bring significantly more efficient \&affordable batteries [7].

\section{CONCLUSION}

In summary we can say that tremendous amount of changes have been made in the past few years in developing a perfect OSCs toward high PCE, long lasting operation, large area processing showing a bright future of applications on a large scale. We expect to see both OSCs \& PCSs bring about substantial changes to the global energy \& environment. It can be said that the evidence of limited global impact of PV is marked by the increasing market of fossil fuels in generating the electricity. We can't deny that OPV technology is in its early stage of development, but considering its withstanding \& promising qualities like ecofriendly, flexibility, vision for mass production, large area production of fabrication which consists of spin coating, vaporization, etc OPV are considered one of the key elements in the source of energy. The review in this paper cites that there is enough room for improvement and further research in the terms of necessary OSCs technological requirements including stability, efficiency, material reliability, performance \& commercialization of OPV solar cell. 


\section{REFERENCES}

[1] Jiang Chuan, Li Tianze, Hou Luan and Zhang Xia, Research on the Characteristics of Organic Solar Cells, Journal of Physics Conference Series, (2011), 276, 012169.

[2] Moritz Riede , Donato Spoltore, Karl Leo, Organic Solar Cells- The Path To Commercial Success, Advanced Energy Materials, (2020), 2002653, 1-10.

[3] Egidius Rutatizibwa Rwenyagila " A Review of Organic Photovoltaic Energy Source and Its Technological Designs" Article ID 1656512| https://doi.org/ $10.1155 / 2017 / 1656512$.

[4] Wu, Y. and Gorder, P.F. (2014) Nature Communications. Published on 3 October 2014
[5] Thomas Kietzke, Merck "Advances in opto electronics" 2007(1687-563X) DOI: 10.1155/2007/40285

[6] Padraig Belton |Technology of Business reporter, "A breakthrough approaches for solar power".

[7] T. Wolfgang, Device Physics of Organic Solar Cells, Dissertation, Technischen Universitat Dresden, Dresden, Germany, (2011). 